\title{
Core and peripheral temperature response to exercise in patients with impaired left ventricular function
}

\author{
M J Griffin, J J O’Sullivan, A Scott, B J Maurer
}

\begin{abstract}
Objective-Exercise induced hypotension is a specific but insensitive indicator of severe coronary artery disease. Skin blood flow is subject to control by baroreceptor mediated reflexes as well as thermoregulatory reflexes. Monitoring skin temperature or the skin to central temperature gradient may be a more sensitive indicator of impaired cardiac output response to exercise than hypotension.
\end{abstract}

Design and participants-Central and skin temperature changes associated with exercise were studied in 10 normal volunteers and eight patients with impaired resting ventricular function due to ischaemic heart disease. Patients exercised according to a modified Bruce protocol. The two sample independent $t$ test was applied to compare the central and peripheral temperatures in the two groups at three minute intervals during exercise and at two minute intervals after exercise.

Results-A significant decrease was found in mean (1 SEM) central temperature on exercise in our patient group $\left(98 \cdot 2(0 \cdot 2)^{\circ} \mathrm{F}\right.$ to $\left.97 \cdot 2(0 \cdot 3)^{\circ} \mathrm{F}\right)$, compared with the normal increase in central temperature $\left(97 \cdot 7(0 \cdot 2)^{\circ} \mathrm{F}\right.$ to $\left.98 \cdot 3(0 \cdot 3)^{\circ} \mathrm{F}\right)$. Mean (1 SEM) skin temperature changes reflected the expected skin blood flow changes with exercise in normal subjects. In the patient group skin temperature declined during exercise $\left(89 \cdot 7(2 \cdot 1)^{\circ} \mathrm{F}\right.$ to $86 \cdot 6(1 \cdot 7)^{\circ} \mathrm{F}$ ) and was significantly lower than normal from six minutes onwards.

Conclusions-The abnormal peripheral temperature changes of patients with impaired resting ventricular function is an early and sensitive indicator of an abnormal haemodynamic response to exercise. It is possible that skin temperature measurement during exercise could help detect exercise induced ventricular dysfunction due to ischaemia or impaired cardiac output due to valvar heart disease.

\section{(Br Heart f 1993;69:388-390)}

The reliable assessment of left ventricular function during exercise is difficult. Monitoring blood pressure with a sphygmomanometer is a technically unsatisfactory, unreliable, and insensitive indicator of left ventricular function during exercise. ${ }^{1}$ Exercise echocardiography or exercise radionuclide scanning are too cumbersome, expensive, or time consuming for routine use.

In normal people exercise increases central temperature, and skin blood flow, after a short initial decrease, increases in response to the rise in core temperature. ${ }^{2}$ In patients with impaired ventricular function, however, skin blood flow decreases with exercise due to sympathetic nervous system overactivity in response to the limited increase in cardiac output.

We tested the hypothesis that the measurement of core and peripheral temperature during exercise would allow for more sensitive detection of an abnormal haemodynamic response to exercise than blood pressure monitoring.

\section{Patients and methods}

We measured simultaneous core (central) and peripheral temperature during exercise testing in 10 normal subjects and eight patients with impaired left ventricular function at rest due to coronary artery disease. Patients belonged to classes 2 and 3 of the New York Heart Association classification. Left ventricular function at rest was assessed by radionuclide scintigraphy or left ventricular angiography within two weeks of the exercise test. Angiography showed significant three vessel disease in all patients. The study was confined to men. Patients whose exercise capacity was limited by angina pectoris or who had sustained a myocardial infarction within the previous three months were excluded.

The subjects were exercised with a modified Bruce protocol. ${ }^{3}$ Blood pressure was measured with a sphygmomanometer and a continous three channel electrocardiogram was recorded. Standard end points for finishing the exercise were used. ${ }^{4}$ Central temperature was measured with an oesophageal thermistor. This was swallowed by the patient to a depth of a quarter of the patient's height and then advanced and retracted to find the point of locally maximum temperature. Such placement makes the temperature recorded relatively insensitive to small displacements of the thermistor. ${ }^{5}$ All subjects tolerated the temperature probe well and all were able to complete the study. Peripheral skin temperature was measured on the middle finger of the left hand. Room temperature was maintained at $22^{\circ} \mathrm{C}$ during all studies. Core and periph-

Departments of
Cardiology,
Physiology, and
Physics, St Vincents
Hospital and
University College
Dublin, Dublin,
Ireland
M J Griffin
J J O’Sullivan
A Scott
B J Maurer
Correspondence to:
Dr Michael Griffin,
Department of Anaesthetics,
St James Hospital, 1 James
Street, Dublin 8, Ireland.
Accepted for publication
6 November 1992.


Figure 1 Central and recovery in normal subjects. temperature (mean (1 SEM)) during exercise

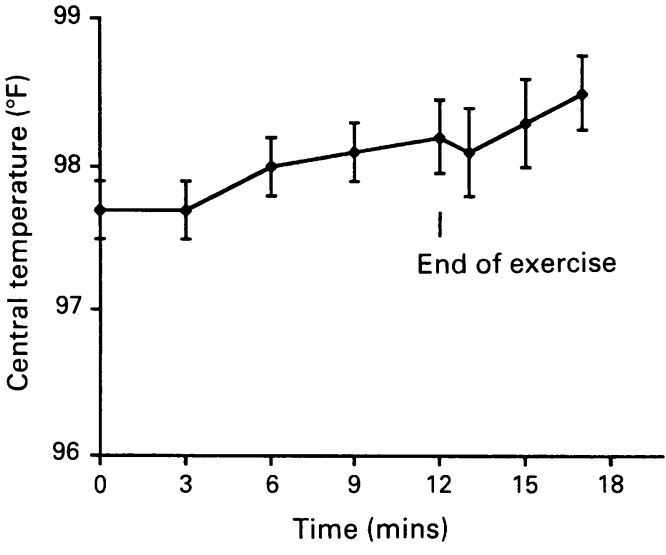

Figure 2 Central temperature (mean (1 SEM)) during exercise and recovery in patients with heart failure.

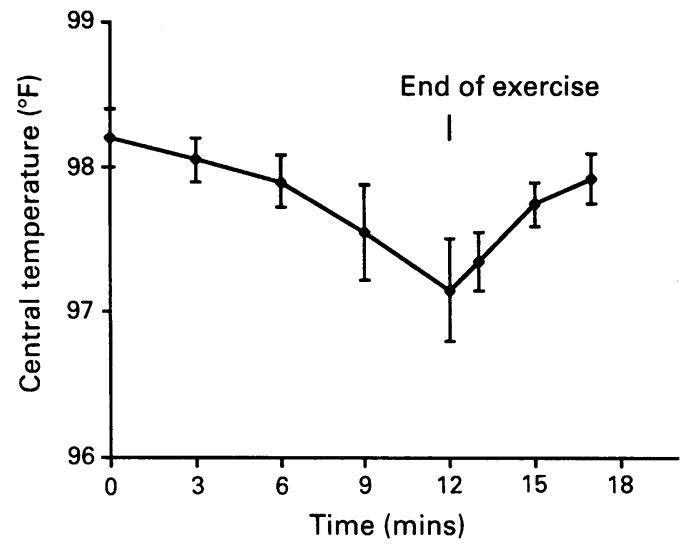

eral temperatures were recorded every $30 \mathrm{sec}$ onds.

STATISTICAL ANALYSIS:

The two sample independent $t$ test was applied to compare the central and peripheral temperatures in the two groups at three minute intervals during exercise and at two minute intervals after exercise.

\section{Results}

The mean age of the 10 normal volunteer subjects was 58.4 (range 52-66) years. The mean age of the group with poor left ventricular function was $62 \cdot 1$ (range 56-70) years. All of these eight patients had had a myocardial infarction more than three months before and all patients were on regular diuretic treatment. Three patients were on ACE inhibitors and no patients were on $\beta$ receptor blocking agents. Poor left ventricular function was due to previous myocardial infarction in all cases.

The table outlines the duration of exercise, heart rate, reason for ending the test, and the peak rise in systolic blood pressure in the eight patients with heart failure. Figure 1 shows the change in central temperature on exercise in the normal group. Mean (SEM) central temperature increased from $97 \cdot 6$ $(0.22)$ to $98 \cdot 4(0.26)^{\circ} \mathrm{F}$ and remained high during the recovery period. Figure 2 shows the central temperature response to exercise in the patient group. Central temperature fell in seven of the eight patients with the mean (SEM) value falling from $98.2(0.2)$ to $97 \cdot 2$ $(0.3)^{\circ} \mathrm{F}(\mathrm{p}<0.05)$. At the end of exercise central temperature was significantly lower in the heart failure group than the normal group $(p<0.05)$. After exercise central temperature gradually increased to the pre-exercise value.

Figure 3 shows the skin temperature response in both groups. Skin temperature increased in the normal group, changing from a mean value of $92.6(0.4)$ to $94.3(0.4)^{\circ} \mathrm{F}$ $(p<0.05)$. In the patients with impaired ventricular function at rest skin temperature decreased with exercise from a mean (SEM) value of $89 \cdot 7(2 \cdot 1)$ to $86 \cdot 6(1 \cdot 7)^{\circ} \mathrm{F}$. Skin temperature was significantly lower in the patient group from six minutes onwards $(p<0.005$ at 12 minutes).

In normal subjects skin temperature shows an initial fall. This is thought to be due to reflex sympathetically induced vasoconstriction that takes place immediately on the initiation of exercise. The peripheral temperature then rises slowly and seems to parallel the increase in central temperature. This centrally mediated peripheral vasodilation is generally believed to occur in an attempt to attenuate the rise in central temperature that takes place with exercise, and this overrides the initial vasoconstriction of the peripheral vessels.

The pattern is significantly different in the patient group. Central temperature actually decreases with exercise in these patients. The initially lower peripheral temperature reflects the compensatory increase in sympathetic tone in patients with impaired ventricular function at rest. Peripheral temperature shows a continuous decrease with exercise as sympathetic tone increases progressively. This takes place as part of the abnormal haemodynamic response to exercise that occurs in patients with impaired ventricular function. Also, the fact that central temperature tends to fall in these patients means that there is no thermoregulatory stimulus for vasodilation to occur, as there is in normal subjects.

Details of exercise test and ejection fraction at rest in eight patients with impaired left ventricular function at rest

\begin{tabular}{llllll}
\hline Patient & $\begin{array}{l}\text { Duration of } \\
\text { exercise (min) }\end{array}$ & $\begin{array}{l}\text { Heart rate } \\
\text { increase } \\
\text { (beats/min) }\end{array}$ & $\begin{array}{l}\text { Reason for } \\
\text { cessation }\end{array}$ & $\begin{array}{l}\text { Maximum increase in } \\
\text { blood pressure }(\mathrm{mm} \text { Hg) }\end{array}$ & $\begin{array}{l}\text { Resting ejection } \\
\text { fraction (\%) }\end{array}$ \\
\hline 1 & 11 & 50 & Fatigue & +20 & 40 \\
2 & 6 & 35 & Dyspnoea & No change & 20 \\
3 & 8 & 10 & Dyspnoea & +30 & 20 \\
4 & 11 & 40 & Dyspnoea & +30 & 28 \\
5 & 11 & 45 & Dyspnoea & +25 & 40 \\
6 & 5 & 80 & Dyspnoea & +15 & 22 \\
7 & 6 & 40 & Dyspnoea & +20 & 29 \\
8 & 8 & 40 & Fatigue & +30 & \\
\hline
\end{tabular}


Figure 3 Skin temperature (mean (1 SEM)) in normal subjects and heart patients during exercise and recovery.

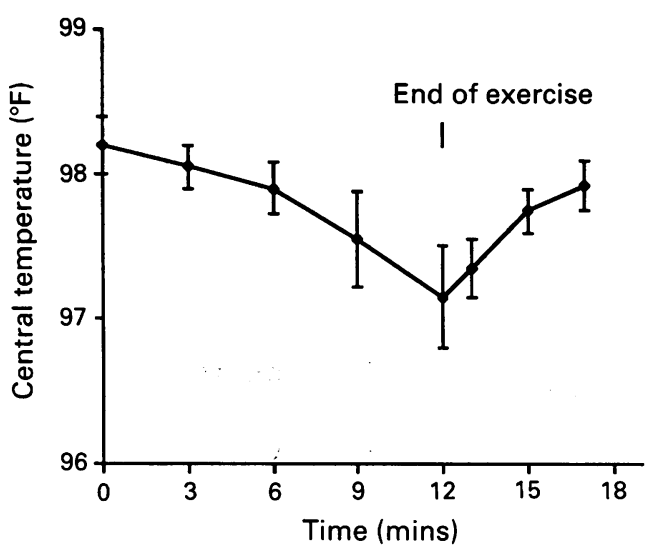

As core temperature falls with increasing exercise in the patient group, measuring the central peripheral gradient did not offer any advantage over studying peripheral temperature changes alone.

\section{Discussion}

A clinically practical and sensitive method of assessing the haemodynamic response to exercise is not yet available. An abnormal blood pressure response to exercise is a specific but very insensitive indicator of severe coronary artery disease ${ }^{16}$ and left ventricular function during exercise. ${ }^{7}$ Furthermore, most physicians are familiar with the difficulties involved in measuring blood pressure in patients undergoing treadmill exercise tests. We have investigated the central and peripheral temperature changes that occur with exercise in normal subjects and patients with impaired ventricular function at rest. Peripheral (skin) temperature directly reflects skin blood flow and shows a characteristic response to exercise. The patients with impaired ventricular function have a noticeably different pattern of peripheral temperature changes. This is because the baroreceptor reflexes and increasing sympathetic tone are predominant influences on skin blood flow in these patients and peripheral blood flow is compromised in an attempt to compensate for the abnormal response of stroke volume to exercise. Also, we now know that central temperature does not increase in such patients and hence the thermoregulatory mediated peripheral vasodilation, known to occur in normal people, is absent in this group. All except one of these patients had a normal blood pressure response to exercise, which confirms that this is not sensitive in detecting poor ventricular performance on exercise. Skin temperature profile was abnor$\mathrm{mal}$ in all these patients indicating that blood pressure is maintained at the expense of perfusion of non-vital organs.

We were surprised to find that central temperature decreased on exercise in the patients with impaired ventricular function. A study with pulmonary artery temperature as an index of core temperature reported similar findings. ${ }^{8}$ It is not easy to explain this fall in temperature but recycling of blood from relatively cool vasoconstricted peripheries and decrease in the central temperature as this blood mixes with the central blood would seem to offer the best explanation. The temperature of blood returning from exercising muscle (femoral vein samples) in patients with heart failure has been measured and found to be consistently lower than core temperature and this would certainly support the theory. ${ }^{9}$ The fact that core temperature does not rise with exercise in the patient group is of some clinical significance. A pacemaker that increases rate with increasing temperature has recently been introduced but the failure of central temperature to rise in our group of patients suggests that they would not derive any benefit from these units. Also, many of the normal subjects showed an initial drop in central temperature at the start of exercise and central temperature was not consistently raised until three minutes after exercise.

The abnormal peripheral temperature response of patients with impaired ventricular function at rest is an early and sensitive indicator of abnormal haemodynamic response to exercise. It remains to be seen whether patients with exercise induced ventricular dysfunction have an abnormal peripheral temperature profile. The ability to detect ischaemia that affects ventricular function and has haemodynamic consequences would be an advance on simply detecting ischaemia as with the routine exercise electrocardiogram. The routine measurement of skin temperature would be a simple and useful adjunct to the standard exercise test and would seem to be much more clinically useful than attempts to monitor blood pressure. The failure of core temperature to rise in patients with decreased ejection fraction may have implications as to the choice of rate responsive pacemakers used in these patients.

1 Hammerstein $\mathrm{K}$, DeRouen $\mathrm{T}$, Dodge $\mathrm{H}$, Zia $M$. Prognostic and predictive value of exertional hypotension in suspected coronary. artery disease. Am $\mathcal{f}$ Cardiol 1977;39:841-8.

2 Zelis R, Mason D, Braunwald E. Partition of blood flow to the cutaneous and muscular beds of the forearm at rest and during exercise in normal subjects and in patients with heart failure. Circ Res 1969;24:799-806.

3 Bruce R, Horsten T. Exercise stress testing in the evaluation of patients with ischaemic heart disease. Prog Cardiovasc Dis 1969;11:371-80.

4 Braunwald E. Heart Disease. A Textbook of Cardiovascula Medicine. Philadelphia: WB Saunders, 1984:8:276.

5 Wenger B, Roberts M, Stolwijk J, Nadel E. Forearm blood flow during body temperature changes produred by leg exercise. $\mathcal{F}$ Appl Physiol 1975;38:58-63.

6 Irving J, Bruce R, DeRouen T. Variations in and significance of systolic pressure during maximal exercise testing. Am 7 Cardiol 1977;39:841-8.

7 Gibbons R, Clements I, Martin H, Zinmeister A, Brown $\mathrm{M}$. Anatomic and functional significance of hypotensive response during supine exercise radionuclide ventricuresponse during supine exercise radio

8 Shellock F, Rubin S, Elldrodt G, Muchlinsk A, Brown H, Swan $\mathrm{H}$. Unusual core temperature decrease in exercising heart failure patients. If Appl Physiol 1983;54 544-50.

9 Shellock F, Swan H, Rubin S. Muscle and femoral vein temperature during short term maximal exercise in hear failure. $\mathcal{F}$ Appl Physiol 1985;58:400-8. 